http://dx.doi.org/10.12775/szhf.2014.009

Tomasz Kupś

\title{
Die erste Versuch, die Kantische Religionsphilosophie in Polen zu verbreiten
}

Im vorliegenden Referat möchte ich nur die wichtigsten Motive der frühesten polnischen Rezeption von Kants Philosophie (zumal der praktischen Philosophie und der Religionsphilosophie) aus dem ersten Jahrzehnt des XIX. Jahrhunderts systematisieren. In erster Linie wird kurz und bündig der Kontext nachgezeichnet, in dem die ersten polnischen Publikationen über Kant erschienen sind (I), danach wird Józef Kalasanty Szaniawski und sein vermeintlicher Kantianismus dargestellt (II). Zum Schluss werden die ausgewählten, an Kants Religionsphilosophie anknüpfenden Motive aus einer Abhandlung von Szaniawski unter dem Titel System des Christentums präsentiert (III).

Noch im XVIII. Jahrhundert dominierte an den polnischen Universitäten die scholastische Philosophie, die am meisten von geistlichen Gelehrten betrieben und gelehrt wurde. Viele Vertreter der neuen philosophischen Strömungen, besonders die Naturwissenschaftler, waren der Meinung, dass diese Art von philosophischer Spekulation völlig an modernen Theorien vorbei gehen und jedes praktischen Wertes beraubt sei. Jede Philosophie, die die empirischen Erkenntnisgründe in Frage stellte oder davon absah, erregte 
Verdacht des religiösen Obskurantismus und wurde zum Ziel von Kritik seitens fortschrittlicher Intellektueller.

Solche Meinungen waren sehr weit verbreitet unter den einflussreichen polnischen Philosophen dieser Zeit. Sowohl die feindliche Grundhaltung gegenüber aller spekulativen Philosophie, vor allem der Einfluß des britischen Empirismus und des französischen Sensualismus (Materialismus), als auch die engen Verbindungen mit der französischen Kultur im allgemeinen, bestärkten die aufklärerischen philosophischen Strömungen.

Die besonders starken Einflüsse der britischen Empiristen und der Hauptvertreter der französischen Aufklärung (Condillac, Buffon) kommen zum Ausdruck in den Schriften der wichtigsten polnischen Philosophen des XVIII. Jahrhunderts, u.a. bei Hugo Kołłątaj, Stanisław Staszic oder Jan Śniadecki.

Die Kritik der philosophischen Spekulation fand viele Anhänger; darunter waren akademische Philosophen (Anioł Dowgrid), Publizisten und Popularisatoren (Tadeusz Czacki), vor allem aber Naturwissenschaftler (wie der schon erwähnte Jan Śniadecki). In den ersten Jahren des XIX. Jahrhunderts setzten sie sich zum Ziel, die neueste Philosophie, auch Kants Philosophie, zu kritisieren. (Man muss an dieser Stelle hinzufügen, dass diese Kritik sich weder auf die Lektüre der Primärliteratur stützte, noch die Vielschichtigkeit der deutschen Philosophie vor und nach Kant berücksichtigte.)

Der schon erwähnte Jan Śniadecki - ein verdienter Mathematiker und Astronom - stellte einen neuen Typus eines weltlichen Philosophen dar, wie wir heute sagen würden, eines Fachwissenschaftlers ${ }^{1}$. Er vertrat die unter den polnischen Naturwissenschaftlern verbreitete Ansicht, dass der wissenschaftliche Fortschritt alle philosophische Spekulation verdrängt habe. Er behauptete, dass in den Schriften der deutschen Philosophen, zumal von Kant und Fichte, wieder eine frühere, im aristotelischen und scholastischen Geiste betriebene, philosophische Spekulation zu Wort kommt. Er stützte sich allerdings nur auf die französische Sekundärliteratur (besonders missverständlich auf Charles Villers [1767-1815] Philosophie de Kant, ou principes fondamentaux de la philolosophie transcendentale [1801], die er offensichtlich völlig falsch verstand ${ }^{2}$ ). Dabei hat er weder Prämissen noch Terminologie der

\footnotetext{
${ }^{1}$ J. Michalski, Z dziejów Towarzystwa Przyjaciół Nauk, Warszawa 1953, S. 52.

${ }^{2}$ Vgl. das Lob Kants zu dieser Schrift: Immanuel Kant an Charles de Villers, v. 15. Aug. 1801: „Herrn Villers danke ich herzlich für Seine Bemühungen, um Ausbreitung einer ächten Philosophie, so wie im Voraus auch, für das mir zugesagte Exemplar Seiner gewiß recht schönen Exposition, und empfehle mich Seiner Freundschaft. I. Kant".
} 
kritischen Philosophie zutreffen erläutert, sondern er betrachtete sie nur als ein Anzeichen für die Rückkehr zur alten Metaphysik ${ }^{3}$. Nur auf diese Weise kann man seine Bewertung verstehen, die er im Jahre 1803 im Briefe an Hugon Kołłątaj äußerte:

Mit Schmerz meines Herzens verstand ich, dass Kants Philosophie, das heißt Aristoteles' Metaphysik aus dem fünfzehnten Jahrhundert, im Neunzehnten Jahrhundert geweckt, die polnischen Köpfe in Warschau infizierte und sie zu verdrehen begann. Ich halte Fichte und Kant für die erbitterten, dunklen und apokalyptischen Köpfe, sie sind Scharlatane neuer Art, die eine Revolution machen wollen..., um die einfachen und klaren Sachen... unter den Gelehrten zu vertreiben. Die Dissertation über Christentum hat mich echt entrüstet, denn außer den dunklen, hochmütigen und sprachverderbenden Ausdrücken fand ich so gewöhnliche Sachen, wie uns Reformanten von der Kanzel hielten ${ }^{4}$.

Die Entrüstung von Śniadecki wurde durch eine Publikation von Józef Kalasanty Szaniawski hervorgerufen, einem damals bekannten Anhänger und Popularistor der deutschen Philosophie, besonders Kants.

Der erwähnte Vortrag von Szaniawski, Das System des Christentums, gehalten auf der Sitzung der Warschauer Gesellschaft der Freunde der Wissenschaften, wurde im Jahre 1803 in den „Annalen der Warschauer Gesellschaft der Freunde der Wissenschaften ${ }^{\text {"5 }}$ veröffentlicht. Auf diese Abhandlung berufe ich mich im letzten Teil meines Artikels.

Józef Kalasanty Szaniawski (1764-1843) ist eine in der polnischen Geschichte der Philosophie sehr ambivalente Person. Ein armer Adliger, der vom Mäzenatentum seiner reichen Freunde profitierte, ein Beamter, ein Bibliophiler und vor allem ein bekannter Freund der deutschen Kuzltur. Ursprünglich arbeitete er als Gerichtskommissar, 1789 trat er der Kavallerie bei. Als er enger mit dem Jakobiner-Kreis verbunden war, verließ er das Militär und engagierte sich bei den Vorbereitungen zum Kościuszko-Aufstand,

\footnotetext{
${ }^{3}$ S. Harassek, Kant w Polsce przed rokiem 1830, Kraków 1916, S. 23.

${ }^{4}$ J. Śniadecki, List do Hugona Kołłątaja, [in:] ders., Pisma filozoficzne, Bd. 2, Warszawa 1958, S. $144-145$.

${ }^{5}$ Dieses bestätigen auch andere, spätere Briefe an Kołłątaj i Czacki (vgl. S. Harassek, Kant $w$ Polsce, S. 24).
} 
nachdem König Stanisław August Poniatowski der Konföderation von Bar beigetreten war.

Nach der Niederlage des Aufstands musste er nach Paris auswandern, wo er eine politische Tätigkeit in der Gesellschaft der Polnischen Republikaner und in der Pariser Deputation (die den militärischen Versuchen, die Unabhängigkeit wiederzuerlangen, und zumal der Legion des Generals Dąbrowski feindlich gesinnt war) fortsetzte. 1801 kehrte er nach Warschau zurück, wo er sich sofort für die wissenschaftliche Arbeit in der Gesellschaft der Freunde der Wissenschaften engagierte. Er war einer der aktivsten Mitglieder dieser Gesellschaft und in Jahren 1802-1804 übte darin die Funktion des Sekretärs aus.

Die Gründung der Gesellschaft der Freunde der Wissenschaften auf dem preußischen Teilungsgebiet war im Jahre 1800 möglich, weil die preußischen Behörden damals eine Politik der Zugeständnisse gegenüber den Polen praktizierten. Szaniawski war ein Loyalist gegenüber den Teilungsmächten und meinte, dass alle Versuche der Veränderung der politischen Lage Polens vergeblich seien. Ganz sicher teilte er die unter den Mitgliedern der Gesellschaft verbreitete Überzeugung:

... dass die Unabhängigkeit Polens zum unwiederbringlich Vergangenen gehört, dass es den Polen nur bleibt, ihre Nationalität durch die Pflege ihrer eigenen Sprache und ihres Kulturerbes zu bewahren, dass sie sich an den Griechen ein Beispiel nehmen sollten, die nach dem Verlust ihrer Unabhängigkeit zu den loyalen Untertanen von Römern wurden und die Eigentümlichkeit ihrer Kultur erhalten haben ${ }^{6}$.

In dieser Lage sollte sich die Gesellschaft zum Ziel setzen, die Nationalsprache und -kultur zu pflegen. Szaniawski hat diese Meinung schon während des Aufenthalts in Paris verfochten, als er seine politische Losung in folgende Worte gefasst hat: „Man muss lernen, aufklären, das Licht verbreiten und nochmals lernen", wodurch er die berühmte Losung von Kościuszko „Man muss kämpfen, schlagen und nochmals schlagen“7 paraphrasierte.

Wir können diese Losungen zumindest teilweise als eigenartige Deklaration „der organischen Arbeit” verstehen, was mit den theoretischen Interessen von Szaniawski (zumal mit der ursprünglich fichteanischen Meinung über

\footnotetext{
${ }^{6}$ J. Michalski, Z dziejów Towarzystwa Przyjaciół Nauk, S. 86.

${ }^{7}$ Ebenda, S. 87.
} 
die Bestimmung des Gelehrten ${ }^{8}$ ) übereinstimmte. Die Schlüsselrolle sollte für ihn die Förderung der geistigen Entwicklung, der Ausbildung und der Sprache und des (polnischen) wissenschaftlichen Wortschatzes spielen. Die wichtigste Aufgabe seines Bildungsprogramms war zweifelsohne die Verbreitung vor allem deutschen Literatur, besonders der philosophischen, mit dem besonderen Nachdruck auf Kants Philosophie.

In den ersten Jahren seiner kulturellen Tätigkeit versuchte er sehr eifrig die deutsche Philosophie in den Vordergrund zu rücken. Er beabsichtigte das breite Publikum für die Philosophie zu interessieren, den Einfluss der französischen, aufklärerischen Ideologen einzuschränken und ihn durch Interesse für den Deutschen Idealismus zu ersetzen. Am Ende der Abhandlung von Szaniawski unter dem Titel Über die moralischen Systeme der Altertümer finden wir die folgende Betrachtung:

Das Schicksal entfernt uns von den politischen Aufgaben, ein natürlicher Trieb der Gemüter versetzt uns in einen neuen Handlungskreis, eine andere Art der Ehre steht uns offen; von der Richtung, die wir diesem Trieb anweisen, wird es abhängen, ob wir die Veränderung ausnutzen werden. Die kämpferischen Völker der Sarmaten (d.h. der Polen) und Germanen wurden einst durch die gemeinsame Angst voneinander getrennt. In unserer Macht ist es zu zeigen, dass beide Völker nur durch die Sprache getrennt, aber durch die Herrschaft der Philosophie eng verbunden sind?

Szaniawski ist von dem Posten des Sekretärs zurückgetreten, als er sich davon überzeugte, dass er der Gesellschaft sein Programm nicht plausibel machen konnte. Eine scharfe Kritik an der französischen Aufklärung war in der Gesellschaft der Freunde der Wissenschaft unmöglich.

Von besonderer Bedeutung für die Gewichtung der wissenschaftlichen Entwicklung Szaniawski's ist eine Chronologie seiner Publikationen, von deren fast alle philosophischen Texte in eine relativ kurze Periode von einigen Jahren nach der Rückkehr aus der Emigration fallen. Es geht um die Jahre 1802-1805 (eventuell bis 1808), d.h. bis zum Anfang der amtlichen Karriere und seiner vollen Hingabe an die politische und Bildungstätigkeit. Die wich-

${ }^{8}$ Vgl. Rozbiór dzieła J. G. Fichtego pt. Einige Vorlesungen über die Bestimmung des Gelehrten (Abhandlung von 1803), Handschrift in Sammlungen der Zielińscy-Bibliothek in Płock, Nr. 498.

${ }^{9}$ O systemach moralnych starożytności, „Roczniki”, Bd. 2, S. 252-253. 
tigsten Jahre sind zweifelsohne die vier Jahre seiner Tätigkeit in der Warschauer Gesellschaft der Freunde der Wissenschaften ${ }^{10}$.

Szaniawski war kein akademischer Lehrer. Er hat keine systematische philosophische Ausbildung erhalten. Seine Ansichten hat er auf dem Wege der selbständigen Forschungen und Interessen gebildet, die nicht durch eine akademische Diskussion gelenkt wurden. Den Historikern der polnischen Philosophie ist er vor allem als Verbreiter der deutschen Philosophie unter den Polen und als „erster polnischer Kantianer“ bekannt. Dieser auf dem Hintergrund der damaligen polnischen Philosophie auffällige Kantanhänger weckte ein großes Interesse unter den Historikern.

Am Anfang des XX Jahrhundert sind viele Publikationen entstanden, die systematisch seine philosophischen Ansichten ${ }^{11}$ rekonstruiert haben, obwohl sie im Grunde genommen keine konsistente Ganzheit bilden. Man muss hinzufügen, dass der Analyse nur die gedruckten Texte von Szaniawski unterzogen wurden und deswegen die Rekonstruktion nicht komplett war. Die wichtigen, unveröffentlicht gebliebenen Handschriften, wurden erst nach dem zweiten Weltkrieg in der Zieliński-Bibliothek in Płock wieder gefunden.

${ }^{10}$ Im Folgenden die Liste der philosophischen Veröffentlichungen aus dieser Zeit:

Co iest filozofja, Warszawa 1802.

O znamienitszych systemach moralnych starożytności, „Roczniki Warszawskiego Towarzystwa Przyjaciół Nauk” 1803, Bd. II (auch im gleichen Jahr separat herausgegeben).

System Chrystianizmu krótko wyłożony, „Roczniki Warszawskiego Towarzystwa Przyjaciół Nauk" 1803, Bd. 2 (auch im gleichen Jahr separat herausgegeben).

Rzut oka na dzieje filozofji od czasu upadku jej u Greków i Rzymian, aż do epoki odrodzenia nauk, „Roczniki Warszawskiego Towarzystwa Przyjaciół Nauk” 1804, Bd. III, (auch im gleichen Jahr separat herausgegeben mit einer bedeutsamen Beilage u.d.T. O sceptykach moralnych i o szkodliwem dążeniu filozofii XVIII wieku).

Rady przyjacielskie młodemu czcicielowi nauk i filozofi, pragnacemu znaleźć pewniejsza drogę do prawdziwego $i$ wyższego oświecenia, Warszawa 1805 (die zweite Auflage der philosophischen Hauptschrift von Szaniawski wurde im Jahre 1823 in Lwów herausgegeben).

${ }^{11}$ Die wichtigsten Besprechungen der Philosophie von Szaniawski enthalten: Wł. M. Kozłowski, J. K. Szaniawski, [in:] Wiek XIX. Sto lat myśli polskiej, Warszawa 1907, Bd. 2; M. Smolarski, J. K. Szaniawski, przyczynek do charakterystyki, „Biblioteka Warszawa” 1910; M. Straszewski, Dzieje filozoficzne myśli polskiej w okresie porozbiorowym, Kraków 1912; F. Gabryl, Polska filozofia religijna, Warszawa 1913, Bd. 1; S. Harassek, Kant w Polsce przed rokiem 1830, Kraków 1916; Geneza i charakterystyka kantyzmu polskiego, „Przegląd Filozoficzny” 1924; M. Manteufflowa, J. K. Szaniawski. Ideologja i działalność 1815-1830, Warszawa 1936; L. Kasiński, Wpływ Kanta na Szaniawskiego, „Przegląd Filozoficzny” 1939. 
Das Wissen über seine Biographie und sein wissenschaftliches Schaffen wird durch die archivalische Forschung immer mehr bereichert ${ }^{12}$.

Unter den ersten Interpretern vom Szaniawskis Werk dominierte die Überzeugung, dass es sehr eng mit der kritischen Philosophie Kants verbunden sei. Im beträchtlichen Maß beruhte diese Überzeugung auf der Legende über seine vermeintliche Teilnahme an Kants Vorlesungen in Königsberg. Der Hinweis darauf („Er besuchte Kants Vorlesungen ohne Vorbereitungkurse”13) befindet sich im Tagebuch von Łukasz Łubieński, aber er wird durch keine Dokumente bestätigt und man muss ihn eher als eine Legende betrachten, die Szaniawski selbst verbreitet hat. Einen ähnlichen Fehler wiederholte auch Mochnacki, indem er über Szaniawski mit unkritischer Verehrung schrieb:

Es gab bei uns einen Menschen mit großen wissenschaftlichen Kenntnissen in Jura, in politischer Ökonomie, in Verwaltung, ein berühmter Philosoph und Kants Schüler. Niemand in Polen hat mehr neue und alte Bücher gelesen, tiefer gedacht als er. Einige Zeit war er ein Schriftsteller aus Berufung, ein Patriot, eine unserer Leuchte ${ }^{14}$.

Der Ausdruck „einige Zeit” gibt treffend das Wesentliche wieder. Nach der Niederlage des Novemberaufstands im Jahre 1831 engagierte sich Szaniawski für eine für Polen schädliche Tätigkeit und nahm die Arbeit in Herrschaftsstrukturen auf, u.a. als ein Gerichtsmitglied, als Sekretär in der Provisorischen Regierung in Kongresspolen, als Generalkurator, endlich als Zensor. Bis zum Lebensende stand er loyal zur preußischen und dann russischen Teilungsmacht. Er ging in die Geschichte als ein übereifriger Loyalist, besonders der russischen Behörden ein.

Die philosophischen Ansichten von Szaniawski - unabhängig davon, welchen verdienstvollen Wert man ihnen heute zuschreiben könnte - haben sich so deutlich vom Hintergrund der unter Polen herrschenden Mode abgehoben, dass viele Spuren davon in den damaligen Tagebüchern verschiedener

${ }^{12}$ In den 60-er Jahren des 20. Jhs. wurden früher unbekannte philosophische Abhandlungen Szaniawskis entdeckt. In den letzten Jahren hat man auch die Sammlung in Płock um neu aufgefundene Entwurfsmanuskripte bereichert.

${ }^{13}$ Zitiert nach: Wiek XIX. Sto lat myśli polskiej, S. 310.

${ }^{14}$ Maurycy Mochnacki, Powstanie narodu polskiego w r. 1830 i 1831, Poznań 1863, S. 132. Zitiert nach: M. Smolarski, J. K. Szaniawski, przyczynek do charakterystyki. - Zu Mochnacki vgl.: Hildegard Schroeder, Studien über Maurycy Mochnacki mit besonderer Berücksichtigung des deutschen Einflusses, Berlin 1953. 
Autoren erhalten geblieben sind. Aufmerksamkeit schenkten ihm natürlich auch Autoren, die keine Sympathie für Szaniawski empfunden haben. Ich führe nur ein Beispiel an.

In den Tagebüchern von Kajetan Koźmian kann man einen Kommentar zu Szaniawskis Engagement für die Verhandlungen mit Russland während des Wiener Kongresses lesen, aber auch darüber, welche Haltung der Fürst Czartoryski zu ihm hatte ${ }^{15}$. Als im Jahre 1814 Szaniawski mit dem Fürst Czartoryski zum Quartier von Zar Alexander I. fuhr, hatte einer der Augenzeuge folgende Notiz gemacht: „Was macht der arme Fürst mit diesem schweren Pedanten, einem Philosophen, dem „Kancista“, der keinen Geist, keinen Witz, keinen diplomatischen Stil hat ${ }^{\text {"16. }}$. Auf polnisch wurde der boshafte Ausdruck „Kancista“ benutzt, was ungefähr heißt - ein Mensch mit Ecken und Kanten, dessen Verhalten anrüchig, eckig, kantig ist. Diese Notiz zeugt somit nicht von einer besonderen Sympathie für Szaniawski und seine philosophischen Präferenzen.

Die Versuche, bei seinen Zeitgenossen eine sachlich tiefere Bedeutung seiner Schriften nachzuweisen, scheinen alle auf Mißvertändnissen zu beruhen (auch wenn man die vor 1960 unbekannten Handschriften berücksichtigt). Man kann ihm aber einige Verdienste für die Verbreitung der deutschen und zumal Kants Philosophie in der dafür ungünstigen Zeit nicht absprechen. Die Schaffensperiode, die auf die Tätigkeit in der Warschauer Gesellschaft der Freunde der Wissenschaften fiel, war zweifelsohne fruchtbar - Szaniawski hat damals sechs Abhandlungen veröffentlicht, viele andere wurden nicht abgeschlossen oder nicht herausgegeben und sind heute nur in den lückenhaften Handschriften zugänglich.

Trotz Ankündigungen stammt aus seiner Feder keine systematische Abhandlung über Kants Philosophie ${ }^{17}$. Szaniawskis Schriften enthalten keine größeren Teile, die eine systematisch bearbeitete Rekonstruktion der kritischen Philosophie bilden würden. Wir finden darin dagegen zahlreiche

${ }^{15}$ Pamiętniki Kajetana Koźmiana obejmujace wspomnienia od roku 1780 do roku 1815, Poznań 1856, Bd. 2, S. 433-434.

${ }^{16}$ Der Bericht ist enthalten in: K. Koźmian, Pamiętniki; angeführt nach: M. Smolarski, J. K. Szaniawski, przyczynek do charakterystyki, S. 144-145.

${ }^{17}$ In Rzut oka na dzieje filozofji od czasu upadku jej u Greków i Rzymian, aż do epoki odrodzenia nauk, 1904, S. 97 befindet sich eine folgende Bemerkung: „Die weitere Geschichte des Skeptizismus gehört nicht hierher; es ist hier nicht der Platz für die Besprechung der letzten und geschickten Entwicklung desselben unter Humes Hand [...] Kant. Ich vertage die Ausführung solcher Art bis zur Darstellung der Philosophie des Königsberger Weisen”. 
Lobsprüche für Kants Philosophie, lockere Anknüpfungen und vereinzelte ungenaue Zitate. Es erhebt sich natürlich die Frage, ob in solchem Kontext Szaniawski wirklich die Rolle zukommt, die ihm noch am Anfang des XX Jahrhunderts in der Geschichte der polnischen Rezeption von Kants Philosophie zugeschrieben wurde?

Um die positive Antwort auf diese Frage zu erhalten, wäre es geboten, einerseits die besondere Lage der polnischen Philosophie am Anfang des XIX Jahrhunderts zu berücksichtigen, andererseits den Charakter das ganzen Schaffens von Szaniawski zu beachten.

II.

Das System chrystyanizmu krótko wyłożony (System des Christentums kurz dargestellt) ist ein Vortrag, der am 5. Mai 1803 auf der Sitzung der Warschauer Gesellschaft der Freunde der Wissenschaften gehalten wurde ${ }^{18}$. Der Text ist aus einigen Gründen hochinteressant.

$\mathrm{Zu} \mathrm{m}$ e in en wird das Christentum darin nicht als Offenbarungsreligion betrachtet, sondern bezeichnet ein Entwicklungsstadium der menschlichen Kultur, nämlich die höchste Stufe des moralischen Fortschritts der Menschheit.

$\mathrm{Zum}$ andere n betont der Autor, dass der Text „eine Fortsetzung der geplanten Vorlesung über moralische Systeme bildet", also des Projekts, das er systematisch seit 1802 in Form von Vorträgen und Publikationen realisiert hat. Man soll an dieser Stelle anmerken, dass eine Fortsetzung der Vorlesungen im Rahmen der religiös konservativen Gesellschaft der Freunde der Wissenschaften unmöglich war. Davon zeugt die Tatsache, dass der Abhandlung u. d. T. Rzut oka na dzieje filozofji od czasu upadku jej u Greków i Rzymian (Umriss der Geschichte der Philosophie seit ihrem Untergang bei Griechen und Römern bis zum Zeitalter der Wiedergeburt der Wissenschaften), die auch in Annalen der Warschauer Gesellschaft der Freunde der Wissenschaften (1804, t. III) erschienen ist, erst in der Buchversion ein Beitrag O sceptykach moralnych i o szkodliwem dążeniu filozofji XVIII wieku (Über moralischen Skeptiker und schädliches Streben der Philosophie des XVIII. Jahrhunderts) beigelegt wurde, der eine Polemik mit den Ansichten der Mitglieder der Gesellschaft enthielt.

${ }^{18}$ Im Druck erschienen im zweiten Band der „Roczniki Towarzystwa Warszawskiego Przyjaciół Nauk" (von 1803). 
$\mathrm{Zu} \mathrm{m}$ dritte $\mathrm{n}$ wurden in der Gesellschaft keine religiösen Themen diskutiert. Vermieden wurden auch die philosophischen Fragen der „spekulativen Philosophie“, wie sie in der Gesellschaft klassifiziert wurden.

Charakteristisch ist die im Titel der Abhandlung annoncierte Absicht, das Christentum als eine systematische Doktrin zu betrachten, die man in einer Linie mit den moralischen Systemen vergangener Philosophie stellen kann. Szaniawski stellt also die Religion der Philosophie nicht gegenüber, sondern er hält sie für die sich einander ergänzenden Geistesäußerungen, in denen man nach demselben Ziel strebt, d.h. nach der moralischen Vervollkommnung der Menschheit. Szaniawski setzt zum einen voraus, dass es eine rationale Doktrin des Christentums gibt, die sich in Form eines systematischen, begrifflichen Systems ausdrücken lässt, und zum zweiten, dass die Menschheit sich moralisch entwickelt, indem sie alle spezifizierbaren Entwicklungsstadien durchläuft, etwa die moralischen Systeme der altertümlichen Philosophen, die jüdische Religion und das Christentum (als das letzte Stadium).

Aus diesem Grunde verteidigt Szaniawski das Recht auf die Betrachtung des Christentums unabhängig von ihrer strikt theologischen bzw. GlaubensPerspektive. Stillschweigend übergeht er die Frage der übernatürlichen Offenbarung, obwohl er ihre Bedeutung für die anfängliche „Gründung“ und „Erweiterung dieser Wissenschaft"19 betont. Zwar behauptet er nicht direkt, dass die Offenbarung instrumentalisiert wurde, aber er lobt ein solches Verhalten.

Szaniawski setzt auch voraus, dass die Christuslehre (in diesem Text benutzt er nicht den Namen Jesu) eine konsistente und mit einem Geist belebte Einheit bildet (deswegen spricht er über den System des Christentums) ${ }^{20}$. Der Philosoph meint, dass derartige einheitliche Auffassungen ihn vor der Verwicklung in theologische Detailstreitigkeiten schützt. Die religiösen Fragen darf man seiner Meinung nach nur dann betrachten, wenn das Christentum nur in den rein moralischen Begriffen interpretiert wird (also ähnlich wie Kant), d.h. bei der Voraussetzung, dass das Christentum einen wesentlichen Beitrag zum Fortschritt der moralischen Entwicklung der Menschheit ausmacht ${ }^{21}$. Es ist wahrscheinlich die wichtigste Kongruenz, die sich hier zu Kant aufdrängt.

\footnotetext{
${ }^{19}$ System chrystyanizmu krótko wyłożony, S. 404-405.

${ }^{20}$ Ebenda, S. 404.

${ }^{21}$ Ebenda, S. 405.
} 
Szaniawski stellt den Zusatand des „Säuglingsalters der Menschheit“, in dem das menschliche Leben sich auf die Befriedigung der einfachen, animalischen Bedürfnisse einschränkt mit dem gegenwärtigen Zustand der „Reife“ zusammen, der von solchen „Genies“ wie Bacon, Kant, Leibniz und Newton repräsentiert wird. Ein Kriterium, das den erwähnte Urzustand der „Unmündigkeit“ von dem Zustand der „Reife/Mündigkeit“ unterscheiden lässt, ist kein im Menschen präsentes theoretisches Vermögen, sondern das Vermögen des „willkürlichen Handelns“.

Im Zustand der moralischen Reife/Mündigkeit kann der Mensch „seine Entwicklung nur von eigenen Bemühungen erhoffen“. Diese Ansicht stimmt mit Kants Idee überein, die der Königsberger Philosoph in Schriften zur moralischen Philosophie und Religionsphilosophie ausgedrückt hat. Ähnlich wie Kant behauptet Szaniawski, dass der moralische Fortschritt im Falle des Einzelmenschen unvermeidlich durch den Tod begrenzt wird und nur im Rahmen der menschlichen Gattung sich endlos entwickeln kann.

Die moralische Entwicklung der Menschheit nennt Szaniawski die „Erziehung" der menschlichen Gattung, die er ausschließlich als den inneren Kampf der höheren mit der niedrigeren Natur des Menschen, d.h. als Kampf zwischen einer dem äußeren Zwang unterworfenen Sinnlichkeit mit der Vernünftigkeit (d.h. dem Vermögen der freien Setzung der Ziele seines eigenen Handelns) $)^{22}$ begreift.

Der Philosoph behauptet, dass der Kampf zwischen dem Guten und Bösen eine "notwendige, vorläufige Etappe“ in der moralischen Entwicklung der Menschheit ausmacht. Ähnlich wie Kant und Schiller ist er der Meinung, dass die Bedeutung dieser Etappe auf der Erziehung des Menschen beruht, dass er nur seinen eigenen Handlungen die Entwicklung seiner Fähigkeiten, besonders moralischer Kompetenzen, zu verdanken hat ${ }^{23}$. Deshalb stellt er nach Kant fest, dass „eine lebhafte und klare Überzeugung uns bewußt macht, dass der sichere Ursprung unserer Willensfreiheit in der Tugend un-

\footnotetext{
22 „Die Geschichte der sittlichen Erziehung unseres Geschlechts bietet uns ein Bild vom fortwährenden Kampf zwischen unteren Vermögen, die uns nicht erlauben, dass wir uns über die Tierklasse emporheben, und den oberen, wo der Keim der menscheneigenen Veredelung steckt. Der Fortschritt der wahren Zivilisation stellt uns einen glücklichen Sieg der oberen Vermögen über die unteren dar." Ebenda, S. 407.

${ }^{23}$ Ebenda, S. 409.
} 
serer Vernunft steckt ${ }^{\text {“24 }}$ und „ein wirklicher moralischer Wert des Menschen sich auf die Handlung stützt, was er niemals vergessen darf “"25.

Daraus stammt Szaniawskis Überzeugung von der Würde des Menschen als eines freien und vernünftigen Wesens. Ähnlich wie Kant, meint er, dass das Wesen dieser Würde (er benutzt ein Wort uzacnienie, was man auf Deutsch ungefähr als „Rechtschaffenheit“ wiedergeben kann) darauf beruht, dass nur der Mensch einen Wert-an-sich-selbst hat und deswegen sich nicht auf die Funktion des bloßen Instruments oder des Mittels zum Erreichen der sogar würdigsten Ziele reduzieren lässt ${ }^{26}$. Szaniawski ist sich mit Kant nicht nur in dieser Frage einig, er wiederholt in der Schrift aus dem Jahre 1805 unter dem Titel Die freundschaftlichen Ratschläge fast wörtlich die Formel aus der Kritik der praktischen Vernunft: „nur der Mensch unter anderen Lebewesen auf der Erde ist für sich selbst ein eigentliches und letztes Ziel ${ }^{“ 27}$.

Die wichtigste Übereinstimmung mit Kants Religionsphilosophie kommt aber zum Ausdruck in der Auffassung der Lehre über das Reich Gottes (das mit der Idee des höchsten Gutes identifiziert wird ${ }^{28}$ ). Man sieht hier deutlich die Ähnlichkeit mit Ausführungen der Dialektik der reinen praktischen Vernunft.

Besondere Aufmerksamkeit verdient, zum einen, die sich wiederholende Anknüpfung an Epikureismus und Stoa, die mit dem Christentum zusammengestellt werden und zum zweiten, die Betonung der Dialektik von Moral und Glückseligkeit sowie Auflösung der Antinomie der praktischen Vernunft mithilfe der Idee des höchsten Gutes:

In dieser Idee vom Reich Gottes finden wir ein vorgestelltes Muster der Welt, die durch solche Lebewesen bewohnt wird, welche das authentische Christentum zu bilden hat. Es sollte eine moralische Welt im ganzen Umfang der Bedeutung sein, die man mit diesem Wort verbinden muss ${ }^{29}$.

${ }^{24}$ J. K. Szaniawski, O znamienitszych systemach moralnych w starożytności, „Roczniki Warszawskiego Towarzystwa Przyjaciół Nauk", Band 2, 1803, S. 198.

${ }^{25}$ J. K. Szaniawski, Co jest filozofia, S. 7.

${ }^{26}$ Diese Bemerkungen betont Szaniawski in seiner Abhandlung zu Fichtes Einige Vorlesungen über die Bestimmung der Gelehrten.

${ }^{27}$ Rady przyjacielskie, S. 95 („In der ganzen Schöpfung kann alles, was man will, und worüber man etwas vermag, auch blos als Mittel gebraucht werden; nur der Mensch und mit ihm jedes vernünftige Geschöpf ist Zweck an sich selbst.“ AA V 87).

${ }^{28}$ AA V 127-128.

${ }^{29}$ System chrystyanizmu krótko wyłożony, S. 428. 
Eine Vorbedingung für das Erreichen „des Rechts auf die Angehörigkeit zur moralischen Welt" ist eine vollständige Übereinstimmung des Willens mit den moralischen Regeln (die Heiligkeit des Willens), „der Zustand der Einwohner der moralischen Welt sollte ein Zustand des Glücks [der Glückseligkeit], ein Zustand der Verwirklichung aller Wünsche sein ${ }^{\text {“30 }}$. Die Heiligkeit des Willens (die Tugend) und die Glückseligkeit, zwei Elemente, die das "höchste Gute“ bilden, bezeichnet Szaniawski ähnlich wie Kant im Kapitel der Kritik der praktischen Vernunft unter dem Titel Von der Dialektik der reinen Vernunft in Bestimmung des Begriffs vom höchsten Gut:

Ohne den ersten [Teil] wäre das höchste Gute kein Gute, weil das moralische Wesen nicht glücklich sein kann, wenn es die Überzeugung entbehrt, dass es des Glücks würdig ist. Ohne den zweiten hätten unsere anfänglichen Schritten auf dem Wege der Entwicklung keine belebende Energie. Der erste Teil bildet eine hinreichende Bedingung und deshalb ist er das Hauptgute, der zweite folgt aus dem ersten und deshalb ist er nur das Nebengute ${ }^{31}$.

Die Heiligkeit bleibt eine Idee, der der Mensch sich nur nähern kann; „die übersinnliche Gnade hilft ihm bei ehrlichen Bemühungen, stärkt seine Schritte" oder, wie Kant sagt, die Gnade gibt dem Menschen das Vertrauen wieder, "daß, wenn wir so gut handeln, als in unserem Vermögen ist, wir hoffen können, daß, was nicht in unserm Vermögen ist, uns anderweitig werde zu statten kommen, wir mögen nun wissen, auf welche Art, oder nicht." 32 .

Aus der Unerreichbarkeit des höchsten Ziels schließt Szaniawski auf die übersinnliche, unbegrenzte Natur des Menschen. Obwohl er dieses Motiv nicht entwickelt, sieht man deutlich, dass er sich die Kritik der praktischen Vernunft zum Vorbild nimmt, wo die Betrachtungen über die Postulate der praktischen Vernunft auf die erwähnten Bemerkungen über die christliche Lehre vom Reich Gottes folgen. Ähnlich wie die Bemerkung über den Zusammenhang zwischen dem erwarteten Glück und dem moralischen Fortschritt: „....in diesem elenden Jammertal kann uns das Glück nicht zuteil werden, obwohl wir durch den ständigen moralischen Fortschritt immer mehr als des Glücks würdig erweisen können“"33 und fügt hinzu „hier

\footnotetext{
${ }^{30}$ Ebenda, S. 429.

${ }^{31}$ Ebenda, S. 450.

${ }^{32}$ AA V 127, Anmerkung.

${ }^{33}$ System chrystyanizmu krótko wyłożony, S. 430-431.
} 
beruhigt das Christentum alle Ansprüche der Vernunft und Bedürfnisse des Herzens, indem er die künftige Welt und dort den allmächtigen Belohner darstellt, der beide Teile, die Heiligkeit und das Glück, die sich in der sinnlichen Welt auf keine notwendige Weise verbinden, mit einem Knoten vereinigt “ ${ }^{\text {"34 }}$. Es ist jedoch nur eine allegorische Vorstellung der moralischen Ordnung, an der der Mensch nur dann teilnehmen kann, wenn er für seine Handlungen die Maximen der praktischen Vernunft zu bestimmen imstande ist: „Das Reich Gottes befindet sich in keiner den Sinnen zugänglichen Stelle, aber es verbirgt sich im In n e ren des Menschen, sucht es dort, dort befinden sich die ehrwürdigen Rechte auf Eure Angehörigkeit zur m or al is chen Welt"35.

In dieser Stelle seiner Argumentation geht der Autor zu einem anderen Thema über, u.a zu Betrachtungen über die metaphysischen Merkmale der Gottheit der jüdischen Religion, die im Christentum um die Idee der Vaterschaft und Liebe bereichert wurden. Im Endeffekt entsteht eine für das Christentum charakteristische Vorstellung der Gottheit ${ }^{36}$. Die Überzeugung über die göttliche Vorsehung bildet eine Grundlage für das Gebot der Gottesliebe („Liebe Gott von ganzem Herzen“).

Die innere Achtung vor dem moralischen Gesetz (und vor seinem Gesetzgeber) verbindet sich hier mit einer äußeren Achtung vor anderen Menschen („Liebe deinen Nächsten wie sich selbst“) - „du solltest ihn lieben, weil du kein anderes Mittel zur Erweisung der Liebe zum Vater hast, als nur durch Erfüllung seines schöpferischen Willens, worauf dein eigenes

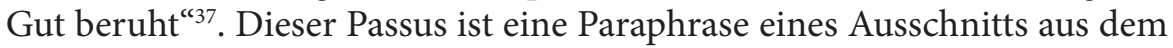
Kapitel Die christliche Religion als natürliche Religion aus dem vierten Teil der Abhandlung Die Religion innerhalb der Grenzen der bloßen Vernunft ${ }^{38}$.

Ähnlich ist auch ein - ungenaues - Zitat auf derselben Seite der Abhandlung von Szaniawski, nur eine Paraphrase aus der Kritik der praktischen Vernunft oder aus der Grundlegung zur Metaphysik der Sitten ${ }^{39}$ ist:

...alle Geschöpfe in der Natur können nacheinander ein Zweck oder ein Mittel sein, der Mensch, insofern er selbst ein vernünftiges Wesen ist, ist ein Zweck an

\footnotetext{
${ }^{34}$ Ebenda, S. 431.

${ }^{35}$ Ebenda, S. 432.

${ }^{36}$ Ebenda, S. 433-434.

${ }^{37}$ Ebenda, S. 435.

${ }^{38}$ AA VI 160.

${ }^{39}$ AA IV 408, V 87, V 131.
} 
sich selbst, niemals bloß ein Mittel und wer auch immer ihn als Mittel benutzt, betrachtet ihn eo ipso als eine passive Maschine und beleidigt seinen erhabenen Charakter ${ }^{40}$.

Das ungenaue Zitat knüpft an den kategorischen Imperativ an. Am Ende zitiert Szaniawski - wieder ungenau - einen Ausschnitt aus der Abhandlung von Reinhold (Briefe über die Kantische Philosophie), „Christus verband auf dem Wege des Herzens die Religion und Moral; jetzt muss man auf dem Wege der Vernunft diese wichtige Verbindung ergänzen und zuverlässig machen " ${ }^{\text {" }}$. Das ist eine zweite Stelle in der Abhandlung von Szaniawski, die eindeutig auf den Kantischen Kontext der Abhandlung über das Christentum hinweist.

Die Absicht von Szaniawski scheint hier eindeutig zu sein - das Christentum führte zwar zur Moralisierung der Religion, aber nicht zur rationalen Begründung dieser Verbindung. Szaniawski musste damals davon überzeugt sein, dass die praktische Philosophie von Kant, besonders seine Religionsphilosophie, eine vernünftige Deutung des Wesens der Religion ausmacht, das in der vollkommensten Form im Christentum verwirklicht wurde.

III.

Die erste polnische Rezeption von Kants Philosophie - es geht hier um Übersetzungen der Schriften und um Notizen und Bearbeitungen der kritischen Philosophie - konzentrierte sich auf die Fragen, die für Polen am interessantesten sein konnten, auch um die politischen Abhandlungen), Aber dies Rezeption führt auch auf die Probleme, die man für die verständlichsten in der Rezeption halten kann (etwa Auszüge aus der ,Anthropologie'). Piotr Chmielowski ${ }^{42}$ - der Autor der ersten polnischen Übersetzung der Kritik der reinen Vernunft - hat festgestellt, dass es auf diese Weise zur Veröffentlichung von Kants Schrift Zur ewigen Frieden und der Ideen zu einer allgemeinen Geschichte in weltbürgerlicher Absicht gekommen sei.

${ }^{40}$ System chrystyanizmu krótko wyłożony, S. 136, Anm. Szaniawski zitiert sehr ungenau. Das sieht man auch in seinen Besprechungen von Vorlesungen Fichtes.

${ }^{41}$ Ebenda, S. 453. Karl Leonhard Reinhold, Briefe über die Kantische Philosophie, 1789, S. 75-76: „Soll nun die Philosophie nach ihrer Art an der Religion thun, was das Christenthum nach der seinigeh an der Moral gethan hat, indem es von Religion zur Moral durch den Weg des Herzens führte, do muß sie von der Moral zur Religion durch den Weg der Vernunft zurückführen; [...]”. Das Zitat von Szaniawski ist nicht wörtlich.

${ }^{42}$ Vgl. S. Harassek, Kant w Polsce. 
Man kann somit richtig vermuten, dass die ersten Übersetzer - Szymon Bielski (im Jahre 1797) und Józef Bychowiec (1799) - „sich Mühe der Übersetzung aus patriotischen Gründen gaben, indem sie in Kants Texten eine

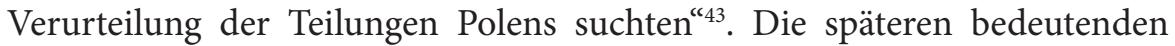
Übersetzungen von Kants Schriften (außer Ausschnitten aus der Anthropologie) sind erst um die Mitte des XIX. Jahrhunderts erschienen.

Der sachliche Gehalt der Abhandlungen von Szaniawski scheint aus der Perspektive der späteren polnischen Bearbeitungen von Kants Philosophie sehr gering zu sein (man kann hier Werke von Bychowiec, Mrongowiusz, Dowgrid, Jaroński und anderen anführen). Es besteht aber kein Zweifel, dass es auf dem Gebiet Polens in den ersten Jahren des XIX. Jahrhunderts außer Szaniawski niemand gab, der den Willen und Mittel hätte, gegen die herrschende Mode, die deutsche Philosophie zu fördern. (Entscheidend war hier wahrscheinlich der Zugang zu der viel gelesenen Zeitschrift und die Mitgliedschaft in der wissenschaftlichen Gesellschaft).

Die Abhandlungen von Szaniawski wurden nicht nur in der schweren politischen Lage Polens veröffentlicht, die für die Verbreitung der deutschen Philosophie ungünstig war, sondern auch bei dem allgemeinen Widerwillen gegen die spekulative Philosophie überhaupt. Vor dem Hintergrund der starken aufklärerischen Strömung sind die Versuche der Verbreitung der Kantischen praktischen Philosophie, zumal der Religionsphilosophie besonders auffällig. In meinem Vortrag wollte ich eben auf diese Pionierarbeiten in Polen hinweisen.

\section{Abstract}

The First Attempt of Propagation of Kant's Philosophy in Poland

In this paper, I would like only to systematize the most important themes of the earliest Polish reception of Kant's philosophy (especially his practical philosophy and the philosophy of religion) in the first decade of the 19th century. First, I sketch the context of the first Polish publications referring to Kant's philosophy (I), then I present the figure of Joseph Kalasanty Szaniawski's and I refer to the interpretations stating his alleged kantianism (II); at the end, I present selected threads of Szaniawski's entitled "The System of Christianity", referring to Kant's philosophy of religion (III).

Key words: Immanuel Kant, reception, the 19th century philosophy, Joseph Kalasanty Szaniawski, Christian philosophy

${ }^{43}$ M. Żelazny, Referat Piotra Chmielowskiego „Najdawniejsze wiadomości o E. Kancie w piśmiennictwie naszem”, „Ruch Filozoficzny” 2005, S. 58. 